\title{
No Difference in Myosin Kinetics and Spatial Distribution of the Lever Arm in the Left and Right Ventricles of Human Hearts
}

\author{
Divya Duggal ${ }^{1}$, S. Requena ${ }^{1}$, Janhavi Nagwekar ${ }^{1}$, Sangram Raut ${ }^{2}$, Ryan Rich ${ }^{3}$, \\ Hriday Das ${ }^{4}$, Vipul Patel ${ }^{1,5}$, Ignacy Gryczynski ${ }^{1}$, Rafal Fudala ${ }^{1}$, Zygmunt Gryczynski ${ }^{2}$, \\ Cheavar Blair ${ }^{6}$, Kenneth S. Campbell ${ }^{6}$ and Julian Borejdo ${ }^{\text {* }}$ \\ ${ }^{1}$ Department of Cell Biology and Center for Commercialization of Fluorescence Technologies, University of North Texas, \\ Health Science Center, Fort Worth, TX, United States, ${ }^{2}$ Department of Physics and Astronomy, Texas Christian University, \\ Fort Worth, TX, United States, ${ }^{3}$ Department of Mathematics and Physics, Texas Wesleyan University, Fort Worth, TX, United \\ States, ${ }^{4}$ Center for Neuroscience Discovery, Institute for Healthy Aging, University of North Texas, Health Science Center, \\ Fort Worth, TX, United States, ${ }^{5}$ Center of Emphasis in Diabetes and Metabolism, Paul L. Foster School of Medicine, Texas \\ Tech University Health Sciences Center El Paso, El Paso, TX, United States, ${ }^{6}$ Department of Physiology, University of \\ Kentucky, Lexington, KY, United States
}

OPEN ACCESS

Edited by:

Andrzej Stasiak,

University of Lausanne, Switzerland

Reviewed by:

Maike Krenz,

University of Missouri, United States

Charles Redwood

University of Oxford, United Kingdom

*Correspondence:

Julian Borejdo

Julian.Borejdo@unthsc.edu

Specialty section:

This article was submitted to

Biophysics,

a section of the journal

Frontiers in Physiology

Received: 06 June 2017 Accepted: 08 September 2017

Published: 13 October 2017

Citation:

Duggal D, Requena S, Nagwekar J,

Raut S, Rich R, Das H, Patel V,

Gryczynski I, Fudala R, Gryczynski Z,

Blair C, Campbell KS and Borejdo J

(2017) No Difference in Myosin

Kinetics and Spatial Distribution of the

Lever Arm in the Left and Right

Ventricles of Human Hearts.

Front. Physiol. 8:732

doi: 10.3389/fphys.2017.00732
The systemic circulation offers larger resistance to the blood flow than the pulmonary system. Consequently, the left ventricle (LV) must pump blood with more force than the right ventricle (RV). The question arises whether the stronger pumping action of the LV is due to a more efficient action of left ventricular myosin, or whether it is due to the morphological differences between ventricles. Such a question cannot be answered by studying the entire ventricles or myocytes because any observed differences would be wiped out by averaging the information obtained from trillions of myosin molecules present in a ventricle or myocyte. We therefore searched for the differences between single myosin molecules of the LV and RV of failing hearts In-situ. We show that the parameters that define the mechanical characteristics of working myosin (kinetic rates and the distribution of spatial orientation of myosin lever arm) were the same in both ventricles. These results suggest that there is no difference in the way myosin interacts with thin filaments in myocytes of failing hearts, and suggests that the difference in pumping efficiencies are caused by interactions between muscle proteins other than myosin or that they are purely morphological.

Keywords: cross-bridge orientation, heart ventricles, fluorescence polarization

\section{INTRODUCTION}

In terms of morphology and function whole ventricles are bilaterally different. Smaller force is necessary to pump blood into the pulmonary circulation by the right ventricle than into the systemic circulation by the left ventricle. It is conventionally thought that this difference arises from the dissimilarities of basic fiber structures of both ventricles or that differences arise already at the myocyte level (Belin et al., 2011). Transverse fibers in the free wall of the right ventricle (RV) are shared with oblique fibers in its inter-ventricular septum. The left ventricle (LV) is composed largely of oblique and circumferential fibers (Schwarz et al., 2013), which are known to be more mechanically efficient than the transverse fibers of RV (Sallin, 1969; Austin, 2007). The idea that morphological differences are responsible for functional differences has been reinforced by the fact that there have been no confirmed reports of differences of the heavy chain 
composition or conformations between $\beta$-myosin molecules in the LV and the RV. Further, myosin chains are expressed from the same genes (MYH6 for myosin $\beta$-isoform of ventricular Heavy Chain in humans, MYL6 for Myosin Essential Light Chain, MYL2 for Myosin Regulatory Light Chain, MYL3 for Myosin Alkali Light Chain) (Park et al., 2011; Huang et al., 2015).

However, the question of stronger pumping action of the LV vs. RV cannot be fully assigned to morphological differences until the alternative possibility, that the differences are due to a more efficient function of myosin or other myocyte proteins in the LV than the RV, is answered. The focus of this study is to test whether myosin function is he same or different in both ventricles of failing heart.

The question of stronger pumping action of the LV vs. RV can't be answered by investigating the whole ventricles because whole ventricles (or papillary muscles) contain a large number of myosins $\left(10^{11}-10^{13}\right)$ (Bagshaw, 1982). Data obtained from such a large number gets averaged out, and all the kinetic information, i.e., the rate constants of the mechanochemical cycle of myosin molecule, is lost. In such experiments, the contribution of an individual myosin molecule has to be recognized and not averaged out (Elson, 2004, 2011). Similarly, if a large number of molecules are examined concurrently, the final distribution of myosin will be perfect Gaussian, irrespective of whether the data are taken from the left or right ventricle (Central Limit Theorem, Bracewell, 1965). Because of these technical difficulties, the question of whether individual actin and/or myosin molecules function in a different way in LV and RV has never been asked. Our ability to study a few molecules out of trillions present in a whole ventricle makes the question possible to answer. The object of our study, myosin, is particularly convenient target for investigation because its function can be characterized by the motion of its lever arm that is easily fluorescently labeled In-situ without any damage to its ATPase activity.

The data presented here was obtained from the ventricles from failing hearts. The reason for this is that failing ventricles are relatively easy to obtain (from patients undergoing heart transplant). While failing ventricles suffered variable amounts of damage, myocytes shortened after addition of contracting solution (Figure S2) and retained ATPase activity.

We measured the kinetics and distribution of few myosin molecules in the A-band of an isometrically contracting sarcomere. The A-band is a volume where a force-producing interaction between actin and myosin molecules takes place. Since molecular crowding plays an important role in in-vivo situations (especially in muscle where protein concentration is extremely high, Bagshaw, 1982), such measurements must be carried out under conditions as closely as possible resembling the situation in-vivo, in other words In-situ in the working ventricle

\footnotetext{
Abbreviations: ACF, AutoCorrelation Function; EDC, ethyl-3-[3(dimethylamino)-propyl]-carbodiimide; LC, human Essential Light Chain; FCS, Fluorescence Correlation Spectroscopy; HF, Heart Failure Ventricles. Ventricles from the hearts of patients before heart transplant surgery; LV, Left Ventricle; NF, Ventricles from deceased donors who had Non-failing Heart; OV, Observational Volume; PF, Polarization of Fluorescence; RV, Right Ventricle; SD, Standard Deviation; SSFA, Steady State Fluorescence Anisotropy; XB, myosin Cross-Bridge.
}

(Minton, 2001; Mourao et al., 2014). We show that the kinetics and the steady-state distribution of myosin were the same in contracting left and right ventricles from the failing (HF) human heart. The results suggest that there is no difference in the way myosin interacts with thin filaments in ventricles of failing hearts, and that the difference in ventricular function is caused by proteins other than myosin or by morphological differences between ventricles.

\section{Principle of Measurements}

A myosin head consists of a globular head and $\alpha$-helical lever arm. The lever arm is believed to undergo a large "swing" resulting from actin-activated hydrolysis of ATP by the myosin head. The swing is responsible for a relative sliding of myosin and actin containing filaments and force development during contraction. The essential Light Chain (LC) of myosin resides on the lever arm and therefore is a convenient site to monitor the swing (orientation of the lever arm). Steady State Fluorescence Anisotropy (SSFA) is a well-known method to monitor the orientation. Therefore, the values of SSFA in different physiological states of muscle are an indication of orientation changes during XB cycle.

To monitor the orientation of LC during ventricle contraction, LC was expressed in E. coli, labeled with fluorescent dye and exchanged with native ventricular LC. Figure 1 illustrates how the anisotropy (measure of the orientation) of labeled LC reflects the kinetics of a myosin cross-bridge (XB).

To observe a small number of myosin molecules In-situ it is necessary to carry out experiments on the A-band of the isolated myocytes from human ventricles. The A-band is the volume where actin and myosin interact to produce contractile force. Myocyte A-band contains $\sim 10^{4}$ myosin molecules, still too many to obtain kinetic information. To reduce this number, myosin was intentionally labeled inefficiently with fluorescent probe (i.e., only 1 in a 1,000 myosins were labeled). Myocytes were crosslinked with zero-length cross-linker to eliminate any movement during contraction while preserving ATPase activity. Figure 2 explains how the experiments were carried out.

\section{MATERIALS AND METHODS Chemicals and Solutions}

All chemicals were from Sigma-Aldrich (St Louis, MO). The fluorescent dye SeTau-647-mono-maleimide was from SETA BioMedicals (Urbana, IL). The glycerinating solution contained: $50 \%$ glycerol, $150 \mathrm{mM} \mathrm{KCl}, 10 \mathrm{mM}$ Tris- $\mathrm{HCl} \mathrm{pH} \mathrm{7.5,} 5 \mathrm{mM}$ $\mathrm{MgCl}_{2}$, 5 mM EGTA, 5 mM ATP, 1 mM DTT, 2 mM PMSF, and $0.1 \% \beta$-mercaptoethanol. The rigor solution: $50 \mathrm{mM} \mathrm{KCl,} 10 \mathrm{mM}$ Tris- $\mathrm{HCl} \mathrm{pH} 7.5,2 \mathrm{mM} \mathrm{MgCl}_{2}$. The EDTA-rigor solution: 50 $\mathrm{mM} \mathrm{KCl}, 10 \mathrm{mM}$ Tris- $\mathrm{HCl} \mathrm{pH}$ 7.5, 5 mM EDTA. The Ca-rigor solution: $50 \mathrm{mM} \mathrm{KCl,} 10 \mathrm{mM}$ Tris- $\mathrm{HCl} \mathrm{pH} \mathrm{7.5,} 2 \mathrm{mM} \mathrm{MgCl}_{2}, 0.1$ $\mathrm{mM} \mathrm{CaCl}$. The contracting solution: $50 \mathrm{mM} \mathrm{KCl,} 10 \mathrm{mM}$ Tris$\mathrm{HCl} \mathrm{pH} \mathrm{7.5,} 5 \mathrm{mM} \mathrm{MgCl}_{2}, 0.1 \mathrm{mM} \mathrm{CaCl}_{2}, 5 \mathrm{mM}$ ATP, $20 \mathrm{mM}$ creatine phosphate and 10 units $/ \mathrm{ml}$ of $1 \mathrm{mg} / \mathrm{ml}$ creatine kinase. The relaxing solution: $50 \mathrm{mM} \mathrm{KCl,} 10 \mathrm{mM}$ Tris- $\mathrm{HCl} \mathrm{pH}$ 7.5, 5 $\mathrm{mM} \mathrm{MgCl}$, 5 mM ATP, 2 mM EGTA. 


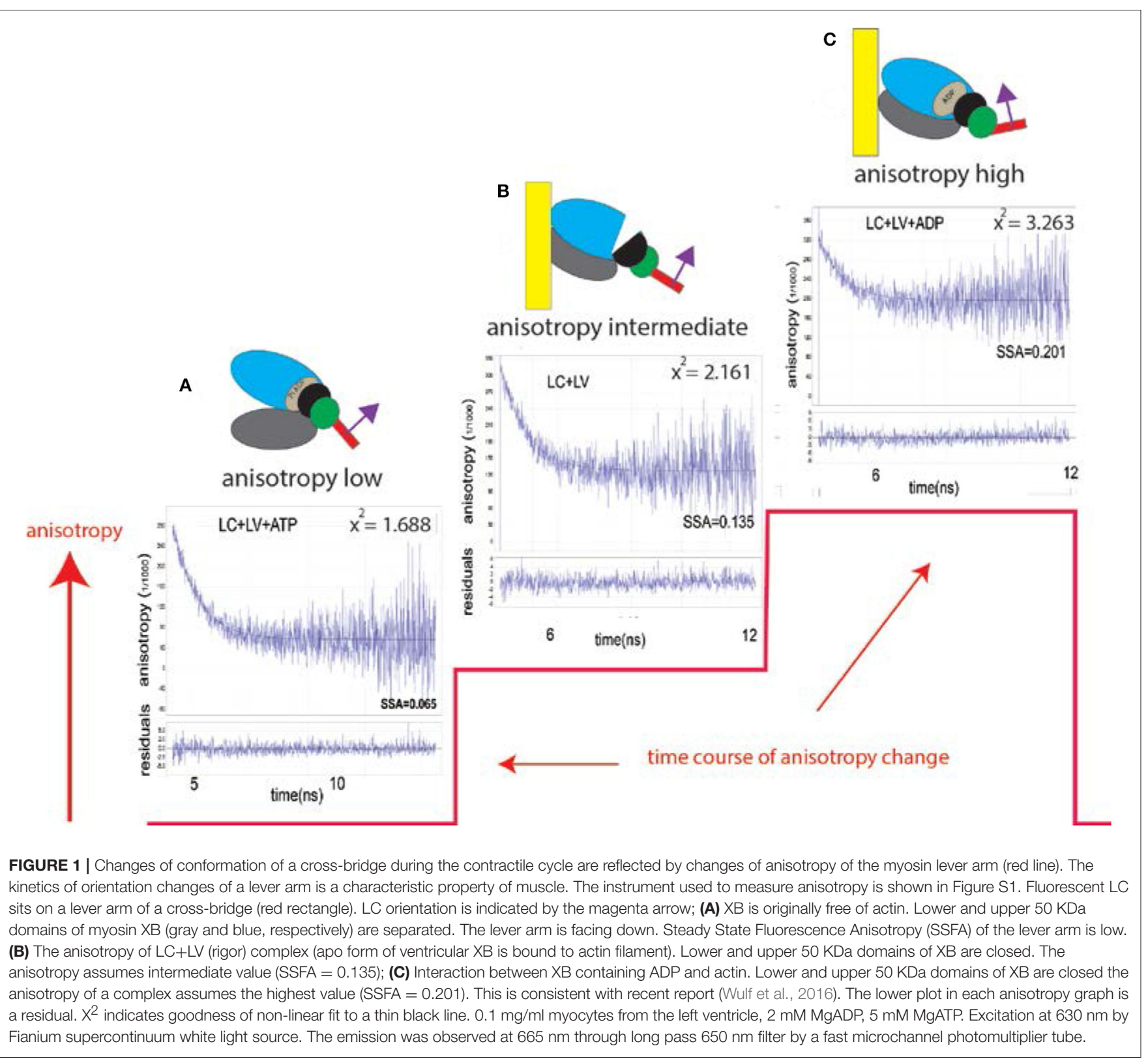

\section{Preparation of Human Essential Light Chain}

LC was genetically modified to contain two thiol groups (rather than 1). This makes it possible to attach two molecules of the dye to the light chain and double the extinction coefficient of the probe. To modify ELC, the human fast skeletal muscle ELC was subcloned into $\mathrm{pQE60}$ vector to produce $\mathrm{pQE}$-ELC expression vector. The LC cDNA insert in PQE60-LC construct contains one Cys at amino acid position 181. We have introduced Cys in place of Gly at amino acid position 174 by site directed mutagenesis to generate pQE60-LCG174C expression vector. The resulting pQE60-LCG174C expression vector contained two Cys residues at amino acid positions 174 and 181. The pQE60LCG174C construct was generated by PCR-based site directed mutagenesis using the QuickChange kit from Strata Gene (La Jolla, CA), pQE60-LC template plasmid, and two complimentary primers:

F-hLCG174C: $5^{\prime}$-gaagtggaagccctgatggcatgtcaagaagactccaatggc $\operatorname{tgc}-3^{\prime}$ and

R-hLCG174C: $5^{\prime}$-gcagccattggagtcttcttgacatgccatcagggcttccact tcttcc-3'. The sequence of the plasmid pQE60-LCG174C was confirmed by DNA-sequencing of both strands of the entire plasmid. Afterwards, the plasmid pQE60-LCG174C was introduced into Escherichia coli M15 cells (Qiagen). The expressed recombinant proteins were purified on Ni-NTAAgarose column and confirmed by immunoblotting with human LC monoclonal antibody. (We also tried to cross-link two thiols with bifunctional rhodamine in order to immobilize the dye, a 
A

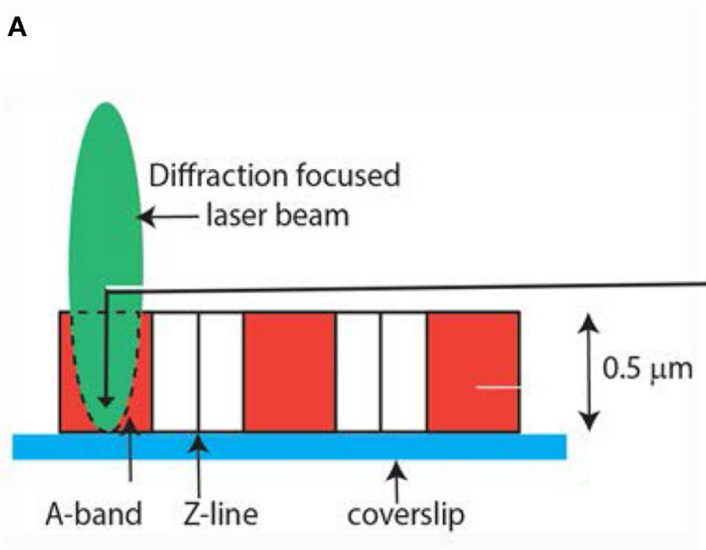

C

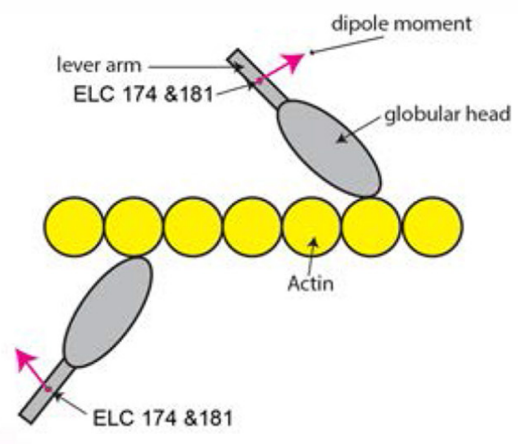

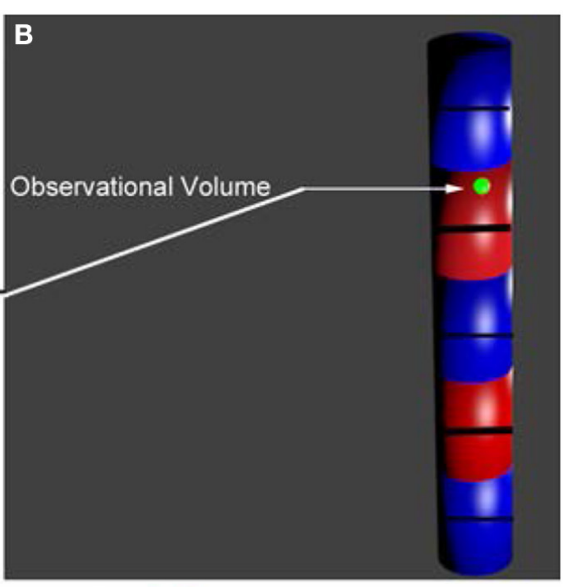

D

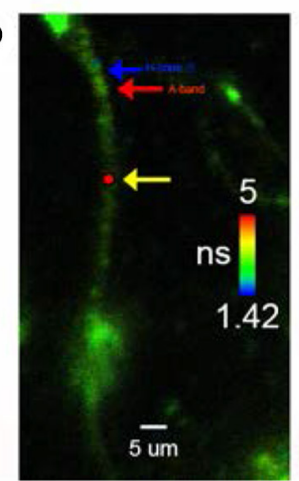

FIGURE 2 | (A) A diffraction limited laser beam (green ellipsoid) is focused on an isolated myocyte sitting on a coverslip. A confocal microscope sees only the Observational Volume (OV, outlined by a broken line). The A-band (red) is the only region where the interaction between actin and myosin occurs. (B) Fluorescence is collected from the OV shown here in $3 \mathrm{D}$ as a green sphere imbedded in the A-band. It is projection of the confocal aperture on the image plane. The diameter of the OV $(0.5 \mu \mathrm{m})$ is equal to the diameter of the confocal pinhole $(50 \mu \mathrm{m})$ divided by the magnification of the objective (100x). Myosin within the A-band is fluorescently labeled (red). The I-bands are non-fluorescent (blue). Thick black lines are the H-zones; thin black lines are the Z-bands. The myocyte contracts (i.e., develops normal force), but does not shorten because it is cross-linked (see Methods); (C) One in approximately one thousand myosin molecules within the thick filament is labeled at LC Cys 174 and Cys 181. The transition dipole moments the dye of two labeled myosins are marked in magenta. Emission dipoles of the dye attached to these two cysteines point in the same direction. Labeling of only 1 in a 1,000 myosins ensures that there are only 6 myosin molecules in the OV (see Methods). Actin monomers (yellow) are non-fluorescent. (D) Fluorescent lifetime image of a myocyte from a non-failing human RV in rigor myocytes were imaged by fluorescent lifetime imaging because a lifetime image is of better quality than a confocal image. Nevertheless, the quality of the image is poor because of intentionally inefficient labeling (see Methods). On the average only $\sim 6$ myosin molecules/half-sarcomere were labeled with the fluorophore (see Methods). The color bar indicates lifetime (in nanoseconds) of a given pixel. The non-fluorescent part ( $\mathrm{H}$-zone) is pointed to by the blue arrow. Data was collected from the red spot (pointed to by the yellow arrow). The data was collected only from the part of a myocyte which was aligned vertically (thus it was not collected from the A-band pointed to by the red arrow). The scale bar is $5 \mu \mathrm{m}$. The red dot (pointed by the yellow arrow) is the 2D projection of the OV. The image has not been contrast enhanced.

procedure originally developed by Irving's group (Corrie et al., 1998, 1999; Hopkins et al., 2002; Lewis et al., 2012), but in our hands this strategy was unsuccessful).

\section{Preparation of Ventricles}

Samples of human myocardium were collected at the University of Kentucky using procedures that were approved by the local Institutional Review Board. The human cardiac samples used in this study were acquired using the collection protocol described by Blair et al. (2016) hearts procured from patients undergoing cardiac transplants at the University of Kentucky and from organ donors who did not have heart failure. Some samples were cut into strips of ventricular walls and analyzed for tension and ATPase by Dr Campbell. Whole ventricles were then given to a researcher immediately after being removed from the patient.
Ventricular tissue was dissected into $\sim 500 \mathrm{mg}$ samples and snapfrozen in liquid nitrogen within $30 \mathrm{~min}$ of being removed from the patient. These samples were then stored in the vapor phase of liquid nitrogen at $-150^{\circ} \mathrm{C}$ until use. The University of Kentucky Institutional Review Board approved all procedures, and subjects gave informed consent (IRB 08-0338-F2L).

All samples were passed to a researcher as soon as they were removed from the patient and snap-frozen in liquid nitrogen within a few minutes. Samples were shipped to UNTHSC on dry ice. Immediately upon arrival in Fort Worth, they were tied to wooden sticks (in a cold room) and placed for $24 \mathrm{~h}$ in glycerinating solution at $0^{\circ} \mathrm{C}$. After $24 \mathrm{~h}$, the glycerinating solution was replaced with a fresh solution and placed at $-20^{\circ} \mathrm{C}$. Myocytes were made from glycerinated hearts after a minimum of 2 weeks at $-20^{\circ} \mathrm{C}$. 


\section{Preparation of Myocytes from a Ventricle}

Human ventricular myocytes were used in all experiments. Use of myocytes eliminates problems associated with the nonuniformity of labeling of sarcomeres and assures better access of ligands to the contractile proteins. Myocytes were prepared from ventricles as follows: ATP present in the glycerinating solution was first removed without causing contraction by washing ventricles three times for $30 \mathrm{~min}$ with ice-cold EDTArigor solution $(50 \mathrm{mM} \mathrm{KCl}, 10 \mathrm{mM}$ Tris- $\mathrm{HCl} \mathrm{pH} \mathrm{7.5,} 5 \mathrm{mM}$ EDTA) followed by extensive washing with the Ca-rigor solution (50 mM KCl, 10 mM Tris- $\mathrm{HCl} \mathrm{pH} 7.5,2 \mathrm{mM} \mathrm{MgCl}, 0.1 \mathrm{mM}$ $\mathrm{CaCl}_{2}$ ). They were homogenized in the Ca-rigor solution (to avoid forming foam upon turbulent stirring when homogenized in EDTA-solution.) in the Cole-Palmer LabGen 125 homogenizer for $10 \mathrm{~s}$ followed by further $10 \mathrm{~s}$ homogenization after a cool down period of $30 \mathrm{~s}$.

\section{ATPase Assay}

The measurements were carried out using Anaspec (Fremont, Ca) Sensolyte MG Phosphate Colorimetric Assay at $30^{\circ} \mathrm{C}$ in a 96-well plate and read on a microplate reader at $650 \mathrm{~nm}$, with and without cross-linker EDC. The myocytes $(1 \mathrm{mg} / \mathrm{ml})$ were incubated in EDC as described above. The reaction mixture of $50 \mathrm{mM} \mathrm{KCl}, 5 \mathrm{mM} \mathrm{MgCl}$, $20 \mathrm{mM}$ EDC, $5 \mathrm{mM}$ ATP in Ca-rigor along with $1 \mathrm{mg} / \mathrm{ml}$ myocytes was made to $80 \mu \mathrm{l}$ in volume to which $20 \mu \mathrm{l}$ Malachite Green (MG) reagent was added. Samples were ran in triplicate. Readings were taken for $30 \mathrm{~min}$ at $2 \mathrm{~min}$. intervals. A graph was plotted to calculate the Pi released per s.

\section{Cross-Linking}

One milligram per milliliter myocytes were incubated for $20 \mathrm{~min}$ at room temperature with $20 \mathrm{mM}$ water soluble cross-linker 1ethyl-3-[3-(dimethylamino)-propyl]-carbodiimide (EDC) in the Ca-rigor solution. It is known that cross-linking of skeletal myocytes does not affect tension or ATPase (Herrmann et al., 1994; Bershitsky et al., 1997; Barman et al., 1998; Tsaturyan et al., 1999). The reaction was stopped by adding $20 \mathrm{mM}$ DTT. To check whether cross-linking does not affect ATPase of cardiac MFs, ATPase was measured independently by two researchers. ATPase varied between $0.028 \pm 0.001$ and $0.032 \pm 0.007$ mols $\mathrm{Pi} / \mathrm{s} / \mathrm{mol}$ myosin, in agreement with (Alpert and Gordon, 1962). 1 In every case $R^{2}$ was 0.98 or 0.99 . The ATPase was slightly higher for the RV than the LV. The $\mathrm{pH}$ of the solution (7.5) remained unchanged throughout the 20 min reaction.

\section{LC labeling}

Recombinant LC (G174C-hLC) was labeled at positions 174 and 181 with three-fold excess of SeTau 647 Maleimide. SeTau647maleimide was chosen to label SH groups on LC because it is excited in the red and hence bypasses most contributions of autofluorescence (Lakowicz, 2006). SeTau is also very resistant to photobleaching (the initial rate of photobleaching was $2.4 \mathrm{~s}^{-1}$ ) because of nano-encapsulation of the squaraine moiety of the dye chromophore system in a mixed aliphatic-aromatic macrocycle. SeTau has a large Stokes shift $(44 \mathrm{~nm})$, very high extinction coefficient (200,000 $\left.\mathrm{M}^{-1} \mathrm{~cm}^{-1}\right)$ and quantum yield (0.65). Its overall fluorescence intensity was 4.2 times larger than the fluorescence intensity of Alexa647. The reaction was allowed to occur overnight. Excess dye was removed by centrifugal filtration followed by overnight dialysis. Using two SeTau molecules per molecule of LC increases overall extinction coefficient to an extraordinary $400,000 \mathrm{M}^{-1} \mathrm{~cm}^{-1}$. It is possible that SeTau resides on two different myosin molecules rather than single myosin, but this is of no consequence because ultimately there are $\sim 6$ molecules of SeTau in the OV (see below). The dyes were wellimmobilized by myocytes in rigor and in the presence of MgADP (see below), and their combined transition dipoles have a welldefined orientation.

\section{Exchange of Labeled LC into Myocyte Myosin}

Following labeling of recombinant peptide with SeTau-647 Maleimide, $5 \mathrm{nM}$ of this protein was exchanged with the native LC of ventricular myosin in the exchange solution containing 15

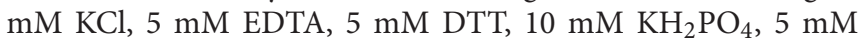
ATP, 1 mM TFP, and 10 mM imidazole, pH 7 (Duggal et al., 2015; Huang et al., 2015). The reaction was allowed to occur at $30^{\circ} \mathrm{C}$ for $10 \mathrm{~min}$. This ensured that the exchange was inefficient (i.e., that only a small fraction of myosin carried fluorescent label). Two milligram per milliliters of freshly prepared myocytes were used.

\section{Data Collection}

The fluorescence was measured by a PicoQuant MT 200 timeresolved fluorescence instrument coupled to an Olympus IX 71 microscope. Before each experiment, the instrument was calibrated by measuring fluorescence of an isotropic solution of a dye with a long fluorescence lifetime (rhodamine 700, excitation at $635 \mathrm{~nm}$ ). Such fluorescence is completely polarized (steadystate anisotropy $r=0.400$, I. Gryczynski, unpublished) because the dye with a long lifetime has no time to reorient itself during a ns short laser pulse. This procedure makes sure that $100 \%$ of emitted light was detected by a parallel $(\|)$ channel. If it was not, the prism position was adjusted until it was. Rotating the birefringent prism by $90^{\circ}$ made sure that the $100 \%$ of emitted light was detected by a perpendicular $(\perp)$ channel. The beam was focused by an Olympus x60, NA $=1.2$ water immersion objective on the A-band of a myocyte. The fluorescence intensity was collected into $10 \mu \mathrm{s}$ bins for $20 \mathrm{~s}$ (i.e., there were 2,000,000 bins). To smooth the data, 1,000 bins were averaged into 2,000 bins yielding overall time resolution of $10 \mathrm{~ms}$. Polarization of fluorescence (PF) and steady-state anisotropy ( $\mathrm{r}$ ) were computed from the orthogonal components of fluorescent light. The autocorrelation function (ACF) and the plot of PF vs. frequency with which it occurs over $20 \mathrm{~s}$ (histogram), was constructed from these 2,000 measurements. Approximately 20-30 myocytes were examined in each ventricle.

\section{Estimating the Number of Observed Myosin Molecules}

To determine the number of observed myosin molecules, it was necessary to measure fluorescence intensity associated with one molecule of SeTau. This number was determined by Fluorescence Correlation Spectroscopy (FCS). The autocorrelation function at delay time 0 of fluctuations of freely diffusing SeTau molecules 
entering and leaving the $\mathrm{OV}$ is equal to the inverse of the number of molecules contributing to the fluctuations $\mathrm{N}=1 / \mathrm{ACF}(0)$ (Magde et al., 1974; Elson, 1985, 2007). Fifteen nanomolars SeTau was illuminated with $1-70 \mu \mathrm{W}$ of laser power at $635 \mathrm{~nm}$. A calibration curve was constructed by plotting the power of the laser vs. the rate of photon arrival per molecule of the dye. From this curve it was determined that the number of photons/s collected from a single SeTau molecule was $\sim 900$.

A typical fluorescent signal obtained from ventricular myocyte is shown in Figure 3.

The average number of observed photons per second in a parallel channel was 2,731 \pm 645 (Figure 3, red). The average number of photons per second in perpendicular channel (black) was $1,161 \pm 364$. The average total fluorescence intensity was $I_{\|}$ $+2 * \mathrm{I}_{\perp}=5,053 \pm 1,373$ counts/s. Therefore, the average number of observed myosin molecules was 5,053/900 $=5.6$. However, it should be noted that as long as the number of molecules is mesoscopic, the exact number does not matter. The same results would have been obtained observing 6 or 60 molecules.

\section{Time Resolved Anisotropy Measurements}

FT300 fluorescence lifetime spectrometer (PicoQuant $\mathrm{GmbH}$, Germany) was used for time-resolved measurements. The muscle was excited by Fianium supercontinuum white light (Fianium Ltd, Whitelase SC400-4). The white light was passed through a monochromator set at $635 \mathrm{~nm}$ at $10 \mathrm{MHz}$ repetition rate. The emission was passed through long pass $650 \mathrm{~nm}$ filter and detected by a fast microchannel photomultiplier tube. The resolution was kept at 4 ps per channel and the pulse width was less than 100 ps. Fluorescence intensity decays were collected while orienting the emission polarizer in vertical and horizontal position respective to the vertically oriented excitation polarizer for measuring anisotropy. The vertical (parallel) and horizontal (perpendicular) intensity decays were used to calculate the time

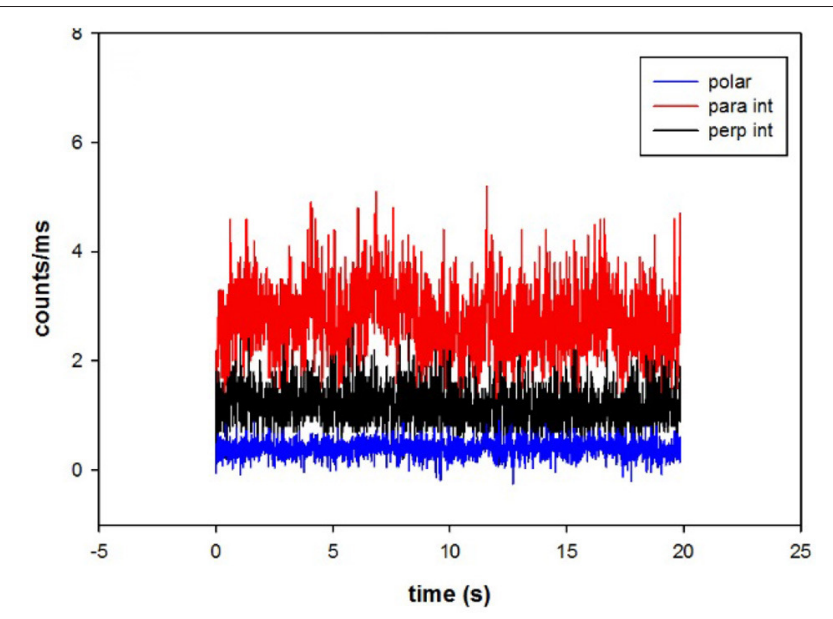

FIGURE 3 | The time course of intensities of fluorescent light from myosin. Fluorescent intensities are polarized parallel (red) and perpendicular (black) with respect to the axis of the myocyte. Polarization of fluorescence is shown in blue. The average polarization of fluorescence was $0.400 \pm 0.158$. The signal from the OV of a left ventricle in the presence of MgADP. dependent anisotropy using the equation $(t)=\left(I \_\right.$Parallel $(t)-$ $I \_$Perpandicular $\left.(t)\right) /\left(I \_P a r a l l e l(t)+2 I \_\right.$Perpandicular $\left.(t)\right)$.

The obtained anisotropy decay was analyzed using Fluofit 4.0 program provided by PicoQuant and was fitted using formula $r(t)=\sum R \_i e^{\wedge}\left(-t / \varnothing \_i\right)$ where $\mathrm{r}$ is the total anisotropy, $\mathrm{R}_{\mathrm{i}}$ is the fractional anisotropy amplitude associated with ith component, $\varnothing \_i$ is the $\mathrm{i}$-th rotation correlation time and $\mathrm{t}$ is the time in nanoseconds. SSFA is the mean of $r$ at all times until the time after which $r$ remains constant (12 ns).

\section{Statistical Analysis}

Comparisons between groups were performed using the unpaired Student's $t$-test by Origin v.8.6 (Northampton, MA). Origin was also used to fit the data by a non-linear routine, calculate histograms, and autocorrelation functions. The significance was defined as $P<0.05$.

\section{Problems}

The chief difficulty in obtaining single molecule data from a ventricle In-situ lies in assuring adequate Signal/Noise ratio. Adequate $\mathrm{S} / \mathrm{N}$ is possible when observing a single myosin molecule in-vitro (Forkey et al., 2003, 2005). However, invitro data cannot be viewed as originating from independent motors (Walcott et al., 2012) (Pate and Cooke, 1991; Baker et al., 2002). In-situ data from a ventricle contains significant contributions from the background. In addition to the very strong autofluorescence due to an extremely dense environment (Bagshaw, 1982), the background consists of a constant fluorescence coming from myosin that is always present in the $\mathrm{OV}$. In contrast to the regular FCS, the myosin molecules do not translate and fluorescent signal does not fluctuate between zero and maximum. In our experiments only the PF fluctuates around the mean. Moreover, to avoid photobleaching the laser beam cannot be focused on the same spot for much more than $20 \mathrm{~s}$.

\section{RESULTS}

\section{Kinetics of Contraction of Failing Ventricles}

We emphasize again that the data presented here was obtained from the ventricles from HF hearts. When cross-linked, they do not shorten, but retain full ATPase activity $0.040 \pm 0.004$ $\mathrm{s}^{-1} / \mathrm{mol} ; 0.034 \pm 0.004 \mathrm{~s}^{-1} / \mathrm{mol}$ for control and cross-linked respectively for the $L V$, and $0.033 \pm 0.003 \mathrm{~s}^{-1} / \mathrm{mol} ; 0.037 \pm 0.004$ $\mathrm{s}^{-1} / \mathrm{mol}$ for control and cross-linked respectively for the RV.

The experiment begins by placing isolated myocyte on an ethanol cleaned cover slip. Because we observe $\sim 6$ myosin molecules (and not a single one), the changes of anisotropy occur gradually (Figure 4, and not in steps as illustrated in Figure 1 which shows changes for a single molecule). The time course of SSFA can be simplified to a scheme shown as a Figure 4, red line. The cycle begins when $\mathrm{XB}$ is dissociated from a thin filament where SSFA is low (A). Binding to a thin filament causes a small increase in SSFA $(B)$ transition $(A \rightarrow B)$. Dissociation of $\mathrm{Pi}$ and assumption of actin-myosin-ADP complex (transition from apoenzyme to holoenzyme $(\mathrm{B} \rightarrow \mathrm{C})$ causes further increase in SSFA (C) in accordance with (Coureux et al., 2004). Finally, 


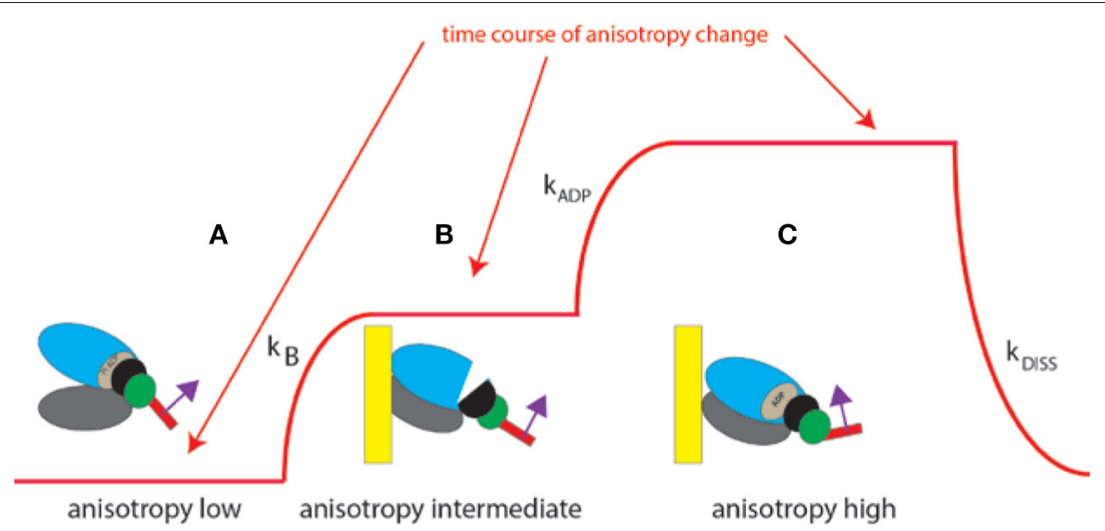

FIGURE 4 | Conformational transitions of 6 XBs during contraction of a ventricle. Red line indicates change of anisotropy. (A) Binding of cross-bridges to a thin filament of a ventricle is characterized by the rate constant $\mathrm{k}_{\mathrm{B}}$; (B) transition from apoenzyme to holoenzyme form of myosin is characterized by the rate constant $\mathrm{k}_{\mathrm{ADP}}$; (C) dissociation of a cross-bridge from a thin filament characterized by the rate constant $\mathrm{k}_{\mathrm{DISS}}$.

dissociation of myosin from actin $(\mathrm{C} \rightarrow \mathrm{A})$ occurs with the rate $\mathrm{k}_{\text {DISS. }}$

To extract the rate constants from fluorescence data we used the method first introduced by Magde and Elson (Magde et al., 1974; Elson, 1985, 2007). In this method Autocorrelation Function (ACF) of fluctuations of anisotropy is calculated. ACF is an average of the sum of products of the instantaneous values of anisotropy and the values of anisotropy delayed by delay time $\tau$ ranging from 0 to $1 \mathrm{~s}$. The process of calculating ACF is shown in Figure S3. The analytical form of ACF of the three-state process is very complex and is fully described in Mettikolla et al. (2011).

A typical example of the ACF data from a HF ventricle is shown in Figure 5. The non-linear fit to the analytical form of ACF is shown as the red line. The experimental data clearly indicates a two-state process. XB binding to actin $\left(\right.$ rate $\left.\mathrm{k}_{\mathrm{B}}\right)$ is too fast to be reflected in the experimental points. This is due to the limited time resolution of the instrument. It collects photons every $10 \mu \mathrm{s}$, but in order to decrease the noise, the $2 \mathrm{M}$ data points are packed in 2,000 bins, 1,000 points per bin, Figure S3. Thus, the instrumental response time is $10 \mathrm{~ms}$. We cannot detect processes faster than $100 \mathrm{~Hz}\left(\mathrm{~s}^{-1}\right)$.

We analyzed data from $6 \mathrm{HF}$ ventricles. One ventricle was from a patient suffering from non-compaction. Data from this ventricle was eliminated. Table 1 shows averages from five remaining ventricles. There was no statistical difference between $\mathrm{LV}$ and $\mathrm{RV}$ for either rate constant. The data from individual ventricles is shown in Figure S4.

\section{Spatial Distribution of XB from Failing Ventricles during Contraction}

A characteristic feature of a distribution of orientations is Full Width at Half Maximum (FWHM) [it is related to Standard Deviation, $S D$; for Gaussian distributions FWHM $=2 \operatorname{Sqrt}(2 \ln 2)$ $=2.355 S D]$. The distribution of polarizations was Gaussian. The differences between FWHM's of polarization of fluorescence of different populations of ventricles are statistically interpretable only when the signal from each half-sarcomere is similar. This

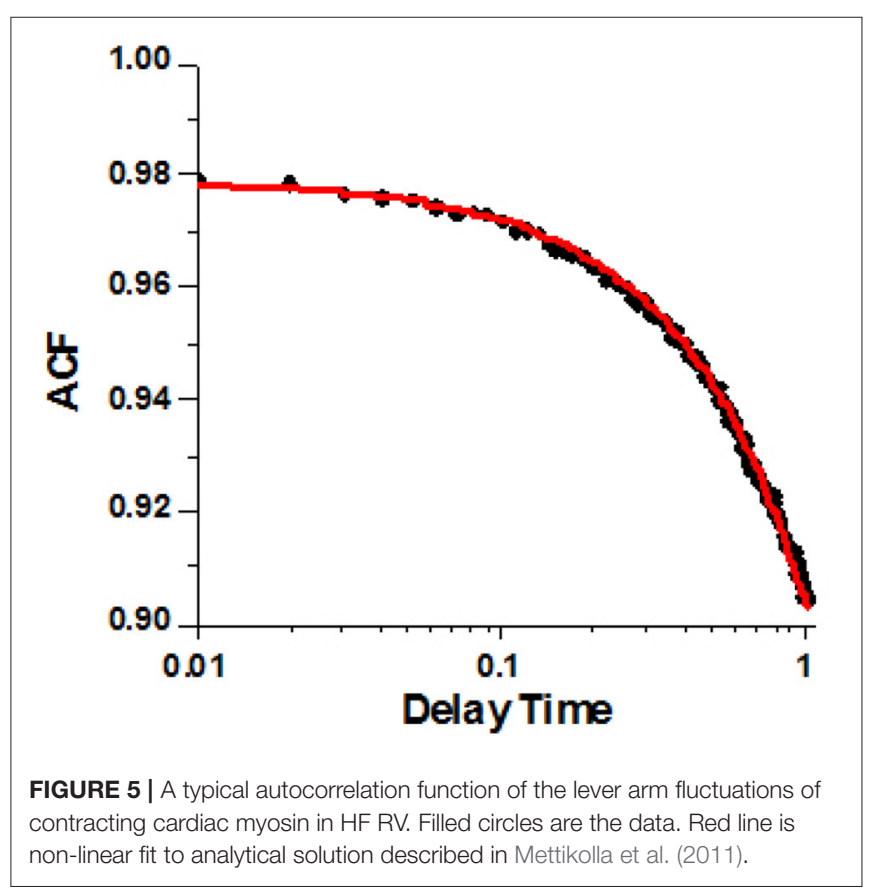

TABLE 1 | Differences between the kinetic rate constants of $L V$ and RV of heart failure ventricles.

\begin{tabular}{lcc}
\hline HF Contraction & $\mathbf{k}_{\text {ADP }}$ & $\mathbf{k}_{\text {DISS }}$ \\
\hline HF LV & $1.69 \pm 0.05$ & $2.24 \pm 0.06$ \\
HF RV & $1.72 \pm 0.04$ & $2.36 \pm 0.20$ \\
\hline
\end{tabular}

The results are averages of 27 experiments on LVs and 26 experiments on RVs. Errors are SD. Overall, the differences in the values of HF LV and RV were not significant.

is because the relative value of FWHM of a strong random signal is small relative to a weak signal (Midde et al., 2013). To comply with this requirement, the power of a laser was adjusted within 1-2 $\mu \mathrm{W}$ of the mean. Examples of measurements 
of 27 experiments from LVs and 26 experiments from RVs are shown in Figure 6. The distributions are displayed here as histograms (plots of frequency of occurrence of a given orientation.

Polarization of Fluorescence $(\mathrm{PF})$ rather than steady-state anisotropy is used here to describe orientation of the lever arm because it has been used routinely to measure conformation of XBs (Dos Remedios et al., 1972; Nihei et al., 1974; Tregear and Mendelson, 1975; Morales, 1984; Hopkins et al., 1998, 2002; Sabido-David et al., 1998). PF is closely related to the SSFA by SSFA $=2 \mathrm{PF} /(3-\mathrm{PF}) . \mathrm{PF}$ is the difference between $\|$ and $\perp$ components of the fluorescent light emitted by the dye, normalized by their sum.

All data is summarized in Table 2. The distributions show no differences in the value of FWHM. $\mathrm{AR}^{2}$ values indicate how well the fitted curve (red) matches a perfect Gaussian (a perfect fit has an $\mathrm{AR}^{2}$ value of 1 ).

\section{DISCUSSION}

We obtained data from a few myosin molecules In-situ in working myocytes. This avoids complications due to the fact that the LV and RV show differences in the basic fiber structures. Further, data was collected under in situ conditions thus taking into account molecular crowding and packing of myosin in thick filaments, which play an important role in crowded systems such as muscle. Taken together, our results suggest that there is no difference in the way myosin interacts with thin filaments in ventricles from HF hearts, and suggests that the difference in pumping efficiencies of ventricles is morphological or that other muscle proteins impose differences: earlier work showed interventricular differences in myocyte function in experimental congestive heart failure of rats. Failing RV and LV myocytes displayed similar decrease in development of maximal force but failing LV myocytes were less $\mathrm{Ca}^{2+}$ sensitive (than failing RV myocytes). There were also differences in expression and activation of PKC- $\alpha$ and in phosphorylation of cTnI and cTnT (Belin et al., 2011). A possible reason for the difference between this work and (Belin et al., 2011) is that different species were examined. Another possibility is that individual molecules were examined here, whereas Belin et al. looked at whole myocytes.

However, the question still remains whether in non-failing (NF) ventricles the difference between LV and RV is also present. In NF ventricles, unlike in $\mathrm{HF}$ ventricles, many differences between whole tissues were observed. Differences have been reported in the ATPase activity (Krug et al., 1987), in the $\alpha$ heavy chain composition and velocity of contraction (Brooks et al., 1987), energy usage (Itoya et al., 1996; Carlsson et al., 2012), amounts of proteins (Cadete et al., 2012), synthesis and degradation of myosin chains (Samarel, 1989), in the contractile performance in dilated cardiomyopathy (McMahon et al., 1996), and congestive heart failure (Belin et al., 2011), in ventricular development (Rosenquist, 1970; de la Cruz et al., 1977; Tam et al., 1997; Mjaatvedt et al., 2001; Buckingham et al., 2005), in the expression of transcription factors expressed during the development (Srivastava et al., 1997) and in the mRNA and miRNA gene expressions (Drake et al., 2011). Our earlier results revealed the functional differences between healthy ventricles in the hearts of mice. (Nagwekar et al., 2014) and rabbits (Nagwekar et al., 2016). This question cannot be answered as yet. We don't have enough molecular data from NF ventricles to make a statistically valid conclusion (Figure S6). However, it must be emphasized that the fact that the existing NF data suggests

TABLE 2 | The FWHM of distribution of lever arm angles of cross-bridges in the A-band of contracting LVs and RVs myocytes from HF human heart.

\begin{tabular}{lcc}
\hline Contraction & FWHM & AR $^{\mathbf{2}}$ \\
\hline HF LV & $0.390 \pm 0.03$ & $0.853 \pm 0.096$ \\
HF RV & $0.381 \pm 0.01$ & $0.889 \pm 0.078$
\end{tabular}

The results are averages of 27 experiments on LVs and 26 experiments on RVs. Errors are SD. By conventional criteria, the statistical significance of difference of FWHM was not statistically significant $(t=0.691, P=0.505)$ within 10 degrees of freedom. $A R^{2}$ measures goodness of fit (closer to 1 the better). The data obtained from individual ventricles is shown in the Figure S5.
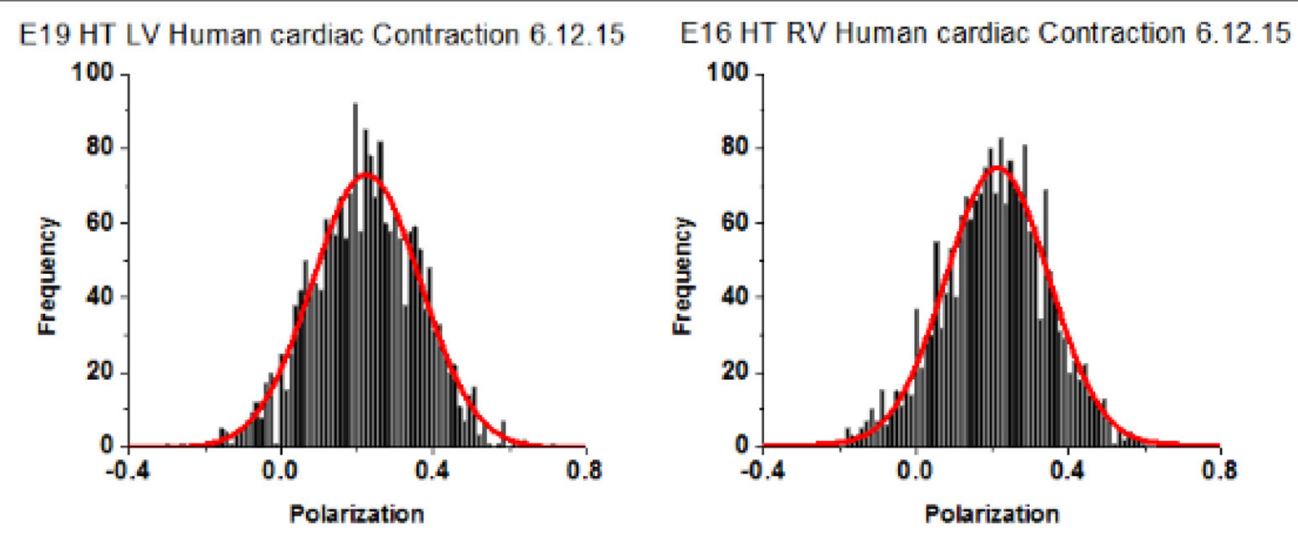

FIGURE 6 | Spatial distribution of lever arm angles of cross-bridges of contracting HF ventricles. The orientation of $\sim 6$ cross-bridges in 27 different preparations of LV and 26 preparations of RV Data shows no difference in FWHM. Bars are data. Red line is the best fit. 
functional differences between healthy ventricles does not imply that lack of differences is somehow normalized by heart failure.

It must be mentioned that the orientation of the lever arm was measured using recombinant light chain exchanged with endogenous LC. This leaves open the possibility that the data may not reflect exactly on the interactions of endogenous LC which carries no fluorophores. Post-translational modifications are unlikely to affect the results because in the mutated clone $\mathrm{G}$ has been shown to have Cys at amino acid position 147 by DNA sequencing. Therefore, expression of this mutated plasmid is expected to produce $\mathrm{G} 147 \mathrm{C}$ isoform of the protein. The most obvious post-translational modification, formation of Cys-Cys link between Cys 147 and 181, is impossible because sample was pre-washed with DTT (1l. 265). The isoform shift will occur upon substituting Gly for Cys and the overall negative charge will decrease.

When we obtain statistically valid results for animal NF ventricles, it will have important clinical implications. We will then be able to make comparisons between LVs and RVs from $\mathrm{HF}$ and NF ventricles. If it turned out that LV from NF hearts are different from LVs from HF hearts or that RVs from NF hearts are different from RVs from HF hearts it would open the possibility of manipulation of rate constants and distributions to make the ventricular function of $\mathrm{HF}$ and $\mathrm{NF}$ ventricles similar. We think that the precise modifiers of rate constants and distributions will be found to minimize the differences between $\mathrm{NF}$ and HF ventricles. Myosin is a particularly convenient target for investigation because it is an allosteric molecule, where the actin binding domain, the nucleotide binding pocket, and the converter domain are in constant communication (Spudich, 2014). It should therefore be possible to find allosteric effectors that modify its function. The binding of a small molecule to myosin may perturb its function, specifically the efficiency of its interaction with actin and thus power output of a ventricle (Spudich, 2014). Any drug that potentiates of power output is likely to have a positive effect on a ventricle pumping too weakly. Likewise, specific inhibitors that affect force development (by altering the rate constants) to reduce power and velocity of muscle shortening are likely to be effective.

\section{REFERENCES}

Alpert, N. R., and Gordon, M. S. (1962). Myofibrillar adenosine triphosphatase activity in congestive heart failure. Am. J. Physiol. 202, 940-946.

Austin, E. H. III. (2007). The ventricular myocardial band of Torrent-Guasp - the controversy: an editorial. Semin.Thorac. Cardiovasc. Surg. Pediatr. Card. Surg. Annu. 10, 87-88. doi: 10.1053/j.pcsu.2007.02.001

Bagshaw, C. R. (1982). Muscle Contraction. London: Chapman and Hall.

Baker, J. E., Brosseau, C., Joel, P. B., and Warshaw, D. M. (2002). The biochemical kinetics underlying actin movement generated by one and many skeletal muscle myosin molecules. Biophys. J. 82, 2134-2147. doi: 10.1016/S0006-3495(02)75560-4

Barman, T., Brune, M., Lionne, C., Piroddi, N., Poggesi, C., Stehle, R., et al. (1998). ATPase and shortening rates in frog fast skeletal myofibrils by timeresolved measurements of protein-bound and free Pi. Biophys. J. 74, 3120-3130. doi: 10.1016/S0006-3495(98)78018-X

Belin, R. J., Sumandea, M. P., Sievert, G. A., Harvey, L. A., Geenen, D. L., Solaro, R. J., et al. (2011). Interventricular differences in myofilament

\section{CONCLUSIONS}

- We looked for the differences between myosin cross-bridges of the LV and RV.

- Experiments were done In-situ thus accounting for molecular crowding.

- Cross-bridges from the left and right ventricles of contracting HF hearts were kinetically identical.

- Cross-bridges from the left and right ventricles of contracting HF hearts were identically distributed in space.

- The difference in pumping efficiencies between LV and RV of HF hearts are caused by muscle proteins other than myosin or are morphological.

\section{AUTHOR CONTRIBUTIONS}

JB formulated the problem, designed experiments, and helped writing the paper. DD and JN carried out experiments. SRa and SRe RF, VP, and RR carried out computations. HD did genetic manipulation of myosin. IG and ZG did spectral analysis. CB and $\mathrm{KC}$ did initial test of the ventricles in Lexington and supplied them to JB.

\section{ACKNOWLEDGMENTS}

We thank Dr. G.E. Taffet (Baylor College of Medicine) for suggestions. This work was supported, in whole or in part, by the National Institutes of Health R01 AR048622 (to JB) and R01HL090786 (to JB and Dr. Danuta Szczesna-Cordary), R01EB12003 (to ZG) and by the Bridge Grant \# RI6127 from UNTHSC.

\section{SUPPLEMENTARY MATERIAL}

The Supplementary Material for this article can be found online at: https://www.frontiersin.org/articles/10.3389/fphys. 2017.00732/full\#supplementary-material

function in experimental congestive heart failure. Pflugers Arch. 462, 795-809. doi: 10.1007/s00424-011-1024-4

Bershitsky, S. Y., Tsaturyan, A. K., Bershitskaya, O. N., Mashanov, G. I., Brown, P., Burns, R., et al. (1997). Muscle force is generated by myosin heads stereospecifically attached to actin. Nature 388, 186-190. doi: 10.1038/ 40651

Blair, C. A., Haynes, P., Campbell, S. G., Chung, C., Mitov, M. I., Dennis, D., et al. (2016). A protocol for collecting human cardiac tissue for research. VAD J. 2:12. doi: 10.13023/VAD.2016.12

Bracewell, R. (1965). The Fourier Transform and Its Applications. New York, NY: McGraw-Hill.

Brooks, W. W., Bing, O. H., Blaustein, A. S., and Allen, P. D. (1987). Comparison of contractile state and myosin isozymes of rat right and left ventricular myocardium. J. Mol. Cell. Cardiol. 19, 433-440. doi: 10.1016/S0022-2828(87)80395-4

Buckingham, M., Meilhac, S., and Zaffran, S. (2005). Building the mammalian heart from two sources of myocardial cells. Nat. Rev. Genet. 6, 826-835. doi: $10.1038 / \operatorname{nrg} 1710$ 
Cadete, V. J., Lin, H. B., Sawicka, J., Wozniak, M., and Sawicki, G. (2012). Proteomic analysis of right and left cardiac ventricles under aerobic conditions and after ischemia/reperfusion. Proteomics 12, 2366-2377. doi: 10.1002/pmic.201100604

Carlsson, M., Heiberg, E., Toger, J., and Arheden, H. (2012). Quantification of left and right ventricular kinetic energy using four-dimensional intracardiac magnetic resonance imaging flow measurements. Am. J. Physiol. Heart Circ. Physiol. 302, H893-H900. doi: 10.1152/ajpheart.00942.2011

Corrie, J. E., Brandmeier, B. D., Ferguson, R. E., Trentham, D. R., KendrickJones, J., Hopkins, S. C., et al. (1999). Dynamic measurement of myosin light-chain-domain tilt and twist in muscle contraction. Nature 400, 425-430. doi: $10.1038 / 22704$

Corrie, J. E., Craik, J. S., and Munasinghe, V. R. (1998). A homobifunctional rhodamine for labeling proteins with defined orientations of a fluorophore. Bioconjug. Chem. 9, 160-167. doi: 10.1021/bc970174e

Coureux, P. D., Sweeney, H. L., and Houdusse, A. (2004). Three myosin V structures delineate essential features of chemo-mechanical transduction. EMBO J. 23, 4527-4537. doi: 10.1038/sj.emboj.7600458

de la Cruz, M. V., Sanchez Gomez, C., Arteaga, M. M., and Arguello, C. (1977). Experimental study of the development of the truncus and the conus in the chick embryo. J. Anat. 123, 661-686.

Dos Remedios, C. G., Millikan, R. G., and Morales, M. F. (1972). Polarization of tryptophan fluorescence from single striated muscle fibers. A molecular probe of contractile state. J. Gen. Physiol. 59, 103-120. doi: 10.1085/jgp.59.1.103

Drake, J. I., Bogaard, H. J., Mizuno, S., Clifton, B., Xie, B., Gao, Y., et al. (2011). Molecular signature of a right heart failure program in chronic severe pulmonary hypertension. Am. J. Respir. Cell Mol. Biol. 45, 1239-1247. doi: $10.1165 / \mathrm{rcmb} .2010-0412 \mathrm{OC}$

Duggal, D., Nagwekar, J., Rich, R., Huang, W., Midde, K., Fudala, R., et al. (2015). Effect of a myosin regulatory light chain mutation K104E on actinmyosin interactions. Am. J. Physiol. Heart Circ. Physiol. 308, H1248-H1257. doi: 10.1152/ajpheart.00834.2014

Elson, E. L. (1985). Fluorescence correlataion spectroscopy and photobleaching recovery. Annu. Rev. Phys. Chem. 36, 379-406. doi: 10.1146/annurev.pc.36.100185.002115

Elson, E. L. (2004). Quick tour of fluorescence correlation spectroscopy from its inception. J. Biomed. Opt. 9, 857-864. doi: 10.1117/1.1779234

Elson, E. L. (2007). Introduction to FCS. Fort Worth, TX: UNT.

Elson, E. L. (2011). Fluorescence correlation spectroscopy: past, present, future. Biophys. J. 101, 2855-2870. doi: 10.1016/j.bpj.2011.11.012

Forkey, J. N., Quinlan, M. E., and Goldman, Y. E. (2005). Measurement of single macromolecule orientation by total internal reflection fluorescence polarization microscopy. Biophys. J. 89, 1261-1271. doi: 10.1529/biophysj.104.053470

Forkey, J. N., Quinlan, M. E., Shaw, M. A., Corrie, J. E., and Goldman, Y. E. (2003). Three-dimensional structural dynamics of myosin $\mathrm{V}$ by single-molecule fluorescence polarization. Nature 422, 399-404. doi: 10.1038/nature01529

Herrmann, C., Lionne, C., Travers, F., and Barman, T. (1994). Correlation of ActoS1, myofibrillar, and muscle fiber ATPases. Biochemistry 33, 4148-4154. doi: 10.1021/bi00180a007

Hopkins, S. C., Sabido-David, C., Corrie, J. E., Irving, M., and Goldman, Y. E. (1998). Fluorescence polarization transients from rhodamine isomers on the myosin regulatory light chain in skeletal muscle fibers. Biophys. J. 74, 3093-3110. doi: 10.1016/S0006-3495(98)78016-6

Hopkins, S. C., Sabido-David, C., van der Heide, U. A., Ferguson, R. E., Brandmeier, B. D., Dale, R. E., et al. (2002). Orientation changes of the myosin light chain domain during filament sliding in active and rigor muscle. J. Mol. Biol. 318, 1275-1291. doi: 10.1016/S0022-2836(02)00189-4

Huang, W., Liang, J., Yuan, C. C., Kazmierczak, K., Zhou, Z., Morales, A., et al. (2015). Novel familial dilated cardiomyopathy mutation in MYL2 affects the structure and function of myosin regulatory light chain. FEBS J. 282, 2379-2393. doi: 10.1111/febs.13286

Itoya, M., Mallet, R. T., Gao, Z. P., Williams, A. G. Jr., and Downey, H. F. (1996). Stability of high-energy phosphates in right ventricle: myocardial energetics during right coronary hypotension. Am. J. Physiol. 271, H320-H328.

Krug, H., Punkt, K., and Bittorf, L. (1987). The higher myosin ATPase activity in the right heart ventricle of the rat, proved by histophotometry. Acta Histochem. 82, 115-119. doi: 10.1016/S0065-1281(87)80063-6
Lakowicz, J. R. (2006). Principles of Fluorescence Spectroscopy. New York, NY: Springer.

Lewis, J. H., Beausang, J. F., Sweeney, H. L., and Goldman, Y. E. (2012). The azimuthal path of myosin V and its dependence on lever-arm length. J. Gen. Physiol. 139, 101-120. doi: 10.1085/jgp.201110715

Magde, D., Elson, E. L., and Webb, W. W. (1974). Fluorescence correlation spectroscopy. II. An experimental realization. Biopolymers 13, 29-61. doi: 10.1002/bip.1974.360130103

McMahon, W. S., Mukherjee, R., Gillette, P. C., Crawford, F. A., and Spinale, F. G. (1996). Right and left ventricular geometry and myocyte contractile processes with dilated cardiomyopathy: myocyte growth and beta-adrenergic responsiveness. Cardiovasc. Res. 31, 314-323.

Mettikolla, P., Calander, N., Luchowski, R., Gryczynski, I., Gryczynski, Z., Zhao, J., et al. (2011). Cross-bridge kinetics in myofibrils containing familial hypertrophic cardiomyopathy R58 mutation in the regulatory light chain of myosin. J. Theor. Biol. 284, 71-81. doi: 10.1016/j.jtbi.2011.06.014

Midde, K., Rich, R., Marandos, P., Fudala, R., Li, A., Gryczynski, I., et al. (2013). Orientation and rotational motion of cross-bridges containing phosphorylated and de-phosphorylated myosin regulatory light chain. J. Biol. Chem. 288:7012. doi: 10.1074/jbc.M112.434209

Minton, A. P. (2001). The influence of macromolecular crowding and macromolecular confinement on biochemical reactions in physiological media. J. Biol. Chem. 276, 10577-10580. doi: 10.1074/jbc.R100005200

Mjaatvedt, C. H., Nakaoka, T., Moreno-Rodriguez, R., Norris, R. A., Kern, M. J., Eisenberg, C. A., et al. (2001). The outflow tract of the heart is recruited from a novel heart-forming field. Dev. Biol. 238, 97-109. doi: 10.1006/dbio.2001.0409

Morales, M. F. (1984). Calculation of the polarized fluorescence from a labeled muscle fiber. Proc. Nat. Acad. Sci. U.S.A. 81, 145-149. doi: $10.1073 /$ pnas.81.1.145

Mourao, M. A., Hakim, J. B., and Schnell, S. (2014). Connecting the dots: the effects of macromolecular crowding on cell physiology. Biophys. J. 107, 2761-2766. doi: 10.1016/j.bpj.2014.10.051

Nagwekar, J., Duggal, D., Rich, R., Fudala, R., Gryczynski, I., Gryczynski, Z., et al. (2016). Differences in the spatial distribution of actin in the left and right ventricles of functioning rabbit hearts. J. Med. Photonics doi: 10.1016/j.medpho.2016.07.001. [Epub ahead of print].

Nagwekar, J., Duggal, D., Rich, R., Raut, S., Fudala, R., Gryczynski, I., et al. (2014). Spatial distribution of actin and mechanical cycle of myosin are different in right and left ventricles of healthy mouse hearts. Biochemistry 53, 7641-7649. doi: 10.1021/bi501175s

Nihei, T., Mendelson, R. A., and Botts, J. (1974). Use of fluorescence polarization to observe changes in attitude of S1 moieties in muscle fibers. Biophys. J. 14, 236-242. doi: 10.1016/S0006-3495(74)85911-4

Park, I., Han, C., Jin, S., Lee, B., Choi, H., Kwon, J. T., et al. (2011). Myosin regulatory light chains are required to maintain the stability of myosin II and cellular integrity. Biochem. J. 434, 171-180. doi: 10.1042/BJ20101473

Pate, E., and Cooke, R. (1991). Simulation of stochastic processes in motile crossbridge systems. J. Muscle Res. Cell Motil. 12, 376-393. doi: 10.1007/BF01738593

Rosenquist, G. C. (1970). Location and movements of cardiogenic cells in the chick embryo: the heart-forming portion of the primitive streak. Dev. Biol. 22, 461-475. doi: 10.1016/0012-1606(70)90163-6

Sabido-David, C., Brandmeier, B., Craik, J. S., Corrie, J. E., Trentham, D. R., and Irving, M. (1998). Steady-state fluorescence polarization studies of the orientation of myosin regulatory light chains in single skeletal muscle fibers using pure isomers of iodoacetamidotetramethylrhodamine. Biophys. J. 74, 3083-3092. doi: 10.1016/S0006-3495(98) 78015-4

Sallin, E. A. (1969). Fiber orientation and ejection fraction in the human left ventricle. Biophys. J. 9, 954-964. doi: 10.1016/S0006-3495(69)86429-5

Samarel, A. M. (1989). Regional differences in the in vivo synthesis and degradation of myosin subunits in rabbit ventricular myocardium. Circ. Res. 64, 193-202. doi: 10.1161/01.RES.64.2.193

Schwarz, K., Singh, S., Dawson, D., and Frenneaux, M. P. (2013). Right ventricular function in left ventricular disease: pathophysiology and implications. Heart Lung Circ. 22, 507-511. doi: 10.1016/j.hlc.2013.03.072

Spudich, J. A. (2014). Hypertrophic and dilated cardiomyopathy: four decades of basic research on muscle lead to potential therapeutic 
approaches to these devastating genetic diseases. Biophys. J. 106, 1236-1249. doi: 10.1016/j.bpj.2014.02.011

Srivastava, D., Thomas, T., Lin, Q., Kirby, M. L., Brown, D., and Olson, E. N. (1997). Regulation of cardiac mesodermal and neural crest development by the bHLH transcription factor, dHAND. Nat. Genet. 16, 154-160. doi: 10.1038/ng0697-154

Tam, P. P., Parameswaran, M., Kinder, S. J., and Weinberger, R. P. (1997). The allocation of epiblast cells to the embryonic heart and other mesodermal lineages: the role of ingression and tissue movement during gastrulation. Development 124, 1631-1642.

Tregear, R. T., and Mendelson, R. A. (1975). Polarization from a helix of fluorophores and its relation to that obtained from muscle. Biophys. J. 15, 455-467. doi: 10.1016/S0006-3495(75) 85830-9

Tsaturyan, A. K., Bershitsky, S. Y., Burns, R., and Ferenczi, M. A. (1999). Structural changes in the actin-myosin cross-bridges associated with force generation induced by temperature jump in permeabilized frog muscle fibers. Biophys. J. 77, 354-372. doi: 10.1016/S0006-3495(99)76 $895-5$
Walcott, S., Warshaw, D. M., and Debold, E. P. (2012). Mechanical coupling between myosin molecules causes differences between ensemble and single-molecule measurements. Biophys. J. 103, 501-510. doi: 10.1016/j.bpj.2012.06.031

Wulf, S. F., Ropars, V., Fujita-Becker, S., Oster, M., Hofhaus, G., Trabuco, L. G., et al. (2016). Force-producing ADP state of myosin bound to actin. Proc. Natl. Acad. Sci. U.S.A. 113, E1844-E1852. doi: 10.1073/pnas.1516598113

Conflict of Interest Statement: The authors declare that the research was conducted in the absence of any commercial or financial relationships that could be construed as a potential conflict of interest.

Copyright (C) 2017 Duggal, Requena, Nagwekar, Raut, Rich, Das, Patel, Gryczynski, Fudala, Gryczynski, Blair, Campbell and Borejdo. This is an open-access article distributed under the terms of the Creative Commons Attribution License (CC BY). The use, distribution or reproduction in other forums is permitted, provided the original author(s) or licensor are credited and that the original publication in this journal is cited, in accordance with accepted academic practice. No use, distribution or reproduction is permitted which does not comply with these terms. 